\title{
On The Existence of Yamabe Gradient Solitons
}

\author{
Yadab Chandra Mandal, Shyamal Kumar Hui* \\ Department of Mathematics, The University of Burdwan \\ Golapbag, Burdwan -713104, West Bengal, India \\ E-mails: myadab436@gmail.com, *skhui@math.buruniv.ac.in \\ *Corresponding author
}

(Received February 14, 2018; Accepted April 8, 2018)

\begin{abstract}
The Yamabe soliton is a special soliton of Yamabe flow obtained by R. S. Hamilton, which was formulated due to Yamabe formula introduced by H. Yamabe in 1960. Recently Cao, Sun and Zhang introduced Yamabe gradient soliton. In this paper, the existence of Yamabe gradient solitons on 4-dimensional Riemannian manifold are ensured by some interesting examples.
\end{abstract}

Keywords- Yamabe gradient soliton, Riemannian manifold.

\section{Introduction}

Let $M$ be a Riemannian manifold of dimension $n(n \geq 3)$ equipped with Riemannian metric $g$ and Riemannian connection $\nabla$. Let $R, S$ and $r$ be the curvature tensor, Ricci tensor and scalar curvature of $\mathrm{M}$ respectively. The notion of Yamabe flow was introduced by Hamilton (Hamilton, 1988; Hamilton, 1989) as a tool for constructing metrics of constant scalar curvature in a conformal class of $M$. The Yamabe flow (Hamilton, 1988) is an evolution equation for metrics on $M$ is

$$
\frac{\partial}{\partial t} g=-r g
$$

In dimension $n=2$, the Yamabe flow is equivalent to the Ricci flow. However, for $n>2$, Yamabe flow and Ricci flows are quite different. A Yamabe soliton is a special soliton of the Yamabe flow that moves by one parameter family of diffeomorphisms generated by a fixed (time-independent) vector field $V$ on $M$ and homothetic. A Yamabe soliton on $M$ is a triplet $(g, V, \sigma)$ such that (Hamilton, 1989)

$$
\frac{1}{2} L_{V} g=(r-\sigma) g
$$

where $L_{V}$ denotes the Lie derivative in the direction of $V \in \chi(M)$ and $\sigma \in I R$. The Yamabe soliton is shrinking, steady and expanding as $\sigma<0, \sigma=0, \sigma>0$ respectively. If $\sigma$ is a smooth function on $M$ then the metric satisfying the above equation is called almost Yamabe soliton. It may be noted that Yamabe solitons coincide with the Ricci solitons for $n=2$ and for $n>2$ they are different. The Yamabe solitons are studied by several authors in different context 
International Journal of Mathematical, Engineering and Management Sciences

Vol. 3, No. 4, 491-497, 2018

https://dx.doi.org/10.33889/IJMEMS.2018.3.4-035

such as (Di Cerbo and Disconzi, 2008; Sharma, 2012; Catino et al., 2012; Calvaruso and Zaeim, 2014; Kundu, 2016; Chen and Deshmukh, 2017; Erken, 2017). If there exists a smooth function $f$ on $M$ such that

$f_{, i j}=(r-\sigma) g_{i j}$

then we call $\left(M^{n}, g, f\right)$ is a Yamabe gradient soliton (Cao et al., 2002). This notion also has been studied in (Shu, 2011; Catino et al., 2012; Hsu, 2012; Huang and Li, 2014). It may be noted that every complete non-trivial Yamabe gradient soliton admits a special global warped product structure with a one dimensional base $(\mathrm{He}, 2011)$. The Yamabe gradient solitons are classified in (Daskalopoulos and Sesum, 2013). In this paper, the existence of Yamabe gradient soliton is ensured by some non-trivial examples.

\section{Preliminaries}

This section deals with some basic formulas, those will be required in the next section.

Let $\left(U ; x^{i}\right), \quad i=1,2, \cdots, n$ be a coordinate chart on $M$. The Christoffel symbols of the Riemannian connection is (Bercu and Postolache, 2011)

$$
\Gamma_{i j}^{k}=\frac{1}{2} g^{k l}\left(\frac{\partial g_{l i}}{\partial x^{j}}+\frac{\partial g_{j l}}{\partial x^{i}}-\frac{\partial g_{i j}}{\partial x^{l}}\right)
$$

and the components of $R, S$ and $\mathrm{r}$ are (Bercu and Postolache, 2011).

$$
R_{i j k}^{l}=\frac{\partial \Gamma_{k i}^{l}}{\partial x^{j}}-\frac{\partial \Gamma_{j i}^{l}}{\partial x^{k}}+\Gamma_{k i}^{r} \Gamma_{j r}^{l}-\Gamma_{j i}^{r} \Gamma_{k r}^{l}, S_{i j}=R_{i l k}^{l}, \quad r=g^{i j} S_{i j .}
$$

Also if $f: M \rightarrow I R$ is a smooth function, then we take (Bercu and Postolache, 2011)

$$
f_{, i}=\frac{\partial f}{\partial x^{i}}, \quad f_{, i j}=\frac{\partial^{2} f}{\partial x^{i} \partial x^{j}}-\Gamma_{i j}^{m} f_{, m}
$$

\section{Examples of Yamabe Gradient Soliton}

Example 3.1. Let $M=\left\{\left(x^{1}, x^{2}, x^{3}, x^{4}\right) \in I R^{4}: x^{3} \neq 0\right\}$ be a manifold endowed with the metric

$$
d s^{2}=g_{i j} d x^{i} d x^{j}=\sinh x^{3}\left[\left(d x^{1}\right)^{2}+\left(d x^{3}\right)^{2}\right]+\left(d x^{2}\right)^{2}+\left(d x^{4}\right)^{2},(i, j=1,2,3,4) .
$$

Then the only non-vanishing components of the Christoffel symbols, curvature tensor, Ricci tensor and scalar curvature are computed in (Shaikh et al., 2008)

$\Gamma_{13}^{1}=\frac{1}{2} \operatorname{coth} x^{3}=\Gamma_{33}^{3}=-\Gamma_{11}^{3}, R_{1331}=-\frac{1}{2 \sinh x^{3}}$, 
International Journal of Mathematical, Engineering and Management Sciences

Vol. 3, No. 4, 491-497, 2018

https://dx.doi.org/10.33889/IJMEMS.2018.3.4-035

$S_{11}=-\frac{1}{2 \sinh ^{2} x^{3}}=S_{33}, r=-\frac{1}{\sinh ^{3} x^{3}} \neq 0$,

and hence this $M$ with the metric $\mathrm{g}$ in (3) is a Riemannian manifold (Shaikh et al., 2008) of nonvanishing scalar curvature. Also it is shown that it is non-conformally flat (Shaikh et al., 2008).

For an arbitrary smooth function $f\left(x^{1}, x^{2}, x^{3}, x^{4}\right)$ on $M$, we compute

$$
\left.\begin{array}{l}
f_{, 11}=\frac{\partial^{2} f}{\partial\left(x^{1}\right)^{2}}+\frac{1}{2} \operatorname{coth} x^{3} \frac{\partial f}{\partial x^{3}}, f_{, 12}=\frac{\partial^{2} f}{\partial x^{1} \partial x^{2}}=f_{, 21}, \\
f_{, 13}=\frac{\partial^{2} f}{\partial x^{1} \partial x^{3}}-\frac{1}{2} \operatorname{coth} x^{3} \frac{\partial f}{\partial x^{1}}=f_{, 31}, f_{, 14}=\frac{\partial^{2} f}{\partial x^{1} \partial x^{4}}=f_{, 41}, \\
f_{, 22}=\frac{\partial^{2} f}{\partial\left(x^{2}\right)^{2}}, f_{, 23}=\frac{\partial^{2} f}{\partial x^{2} \partial x^{3}}=f_{, 32}, f_{, 24}=\frac{\partial^{2} f}{\partial x^{2} \partial x^{4}}=f_{, 42}, \\
f_{, 33}=\frac{\partial^{2} f}{\partial\left(x^{3}\right)^{2}}-\frac{1}{2} \operatorname{coth} x^{3} \frac{\partial f}{\partial x^{3}}, f_{, 34}=\frac{\partial^{2} f}{\partial x^{3} \partial x^{4}}=f_{, 43}, f_{, 44}=\frac{\partial^{2} f}{\partial\left(x^{4}\right)^{2}}
\end{array}\right\}
$$

In view of (6), (1) reduces to the following:

$$
\begin{aligned}
& f_{, 11}=(r-\sigma) g_{11}, \text { i.e. } \frac{\partial^{2} f}{\partial\left(x^{1}\right)^{2}}+\frac{1}{2} \operatorname{coth} x^{3} \frac{\partial f}{\partial x^{3}}=(r-\sigma) \sinh x^{3}, \\
& f_{, 12}=(r-\sigma) g_{12}, \text { i.e. } \frac{\partial^{2} f}{\partial x^{1} \partial x^{2}}=0, \\
& f_{, 13}=(r-\sigma) g_{13}, \text { i.e. } \frac{\partial^{2} f}{\partial x^{1} \partial x^{3}}-\frac{1}{2} \operatorname{coth} x^{3} \frac{\partial f}{\partial x^{1}}=0, \\
& f_{, 14}=(r-\sigma) g_{14}, \text { i.e. } \frac{\partial^{2} f}{\partial x^{1} \partial x^{4}}=0, \\
& f_{, 22}=(r-\sigma) g_{22}, \text { i.e. } \frac{\partial^{2} f}{\partial\left(x^{2}\right)^{2}}=(r-\sigma), \\
& f_{, 23}=(r-\sigma) g_{23}, \text { i.e. } \frac{\partial^{2} f}{\partial x^{2} \partial x^{3}}=0, \\
& f_{, 24}=(r-\sigma) g_{24}, \text { i.e. } \frac{\partial^{2} f}{\partial x^{2} \partial x^{4}}=0, \\
& f_{, 33}=(r-\sigma) g_{33}, \text { i.e. } \frac{\partial^{2} f}{\partial\left(x^{3}\right)^{2}}-\frac{1}{2} \operatorname{coth} x^{3} \frac{\partial f}{\partial x^{3}}=(r-\sigma) \sinh x^{3}, \\
& f_{, 34}=(r-\sigma) g_{34}, \text { i.e. } \frac{\partial^{2} f}{\partial x^{3} \partial x^{4}}=0,
\end{aligned}
$$


International Journal of Mathematical, Engineering and Management Sciences

Vol. 3, No. 4, 491-497, 2018

https://dx.doi.org/10.33889/IJMEMS.2018.3.4-035

$$
f_{, 44}=(r-\sigma) g_{44}, \text { i.e. } \frac{\partial^{2} f}{\partial\left(x^{4}\right)^{2}}=(r-\sigma) \text {. }
$$

From the above equations, it follows that $f$ must be a constant function and $\sigma=r$. This leads to the following:

Theorem 3.1. Let $M$ be a Riemannian manifold endowed with the metric $g$ given in (3). Then (M, $g$ ) is Yamabe gradient soliton having constant potential function.

Example 3.2. Let $M=\left\{\left(x^{1}, x^{2}, x^{3}, x^{4}\right) \in I R^{4}: 0<x^{3}<\frac{\pi}{2}\right\}$ be a manifold endowed with the metric

$$
\begin{aligned}
d s^{2} & =g_{i j} d x^{i} d x^{j} \\
& =e^{x^{1}}\left(d x^{1}\right)^{2}+e^{x^{2}}\left(d x^{2}\right)^{2}+\left(d x^{3}\right)^{2}+\sin ^{2} x^{3}\left(d x^{4}\right)^{2},(i, j=1,2,3,4) .
\end{aligned}
$$

Then the only non-vanishing components of $\Gamma_{i j}^{k}$, the curvature tensor $S_{i j}$ and scalar curvature are (Shaikh et al., 2008)

$$
\begin{aligned}
& \Gamma_{11}^{1}=\frac{1}{2}=\Gamma_{22}^{2}, \quad \Gamma_{44}^{3}=-\sin x^{3} \cos x^{3}, \quad \Gamma_{34}^{4}=\cot x^{3}, \\
& R_{3443}=-\sin ^{2} x^{3}, \quad S_{33}=-1, \quad S_{44}=-\sin ^{2} x^{3}, \quad r=-2 \neq 0 .
\end{aligned}
$$

And consequently, this $M$ equipped with $g$ in (17) is a Riemannian manifold of non-vanishing constant scalar curvature. This is also non-conformally flat Riemannian manifold (Shaikh et al., 2008). Now for an arbitrary smooth function $f\left(x^{1}, x^{2}, x^{3}, x^{4}\right)$ on $M$, we compute

$$
\left.\begin{array}{l}
f_{, 11}=\frac{\partial^{2} f}{\partial\left(x^{1}\right)^{2}}-\frac{1}{2} \frac{\partial f}{\partial x^{1}}, f_{, 12}=\frac{\partial^{2} f}{\partial x^{1} \partial x^{2}}=f_{, 21}, \\
f_{, 13}=\frac{\partial^{2} f}{\partial x^{1} \partial x^{3}}=f_{, 31}, f_{, 14}=\frac{\partial^{2} f}{\partial x^{1} \partial x^{4}}=f_{, 41}, \\
f_{, 22}=\frac{\partial^{2} f}{\partial\left(x^{2}\right)^{2}}-\frac{1}{2} \frac{\partial f}{\partial x^{2}}, f_{, 23}=\frac{\partial^{2} f}{\partial x^{2} \partial x^{3}}=f_{, 32}, f_{, 24}=\frac{\partial^{2} f}{\partial x^{2} \partial x^{4}}=f_{, 42}, \\
f_{, 33}=\frac{\partial^{2} f}{\partial\left(x^{3}\right)^{2}}, f_{, 34}=\frac{\partial^{2} f}{\partial x^{3} \partial x^{4}}-\cot x^{3} \frac{\partial f}{\partial x^{4}}=f_{, 43}, \\
f_{, 44}=\frac{\partial^{2} f}{\partial\left(x^{4}\right)^{2}}+\sin x^{3} \cos x^{3} \frac{\partial f}{\partial x^{3}} .
\end{array}\right\}
$$


International Journal of Mathematical, Engineering and Management Sciences

Vol. 3, No. 4, 491-497, 2018

https://dx.doi.org/10.33889/IJMEMS.2018.3.4-035

By virtue of (20), (1) reduces to the following:

$$
\begin{aligned}
& \frac{\partial^{2} f}{\partial\left(x^{1}\right)^{2}}-\frac{1}{2} \frac{\partial f}{\partial x^{1}}=(r-\sigma) e^{x^{1}}, \\
& \frac{\partial^{2} f}{\partial x^{1} \partial x^{2}}=0, \\
& \frac{\partial^{2} f}{\partial x^{1} \partial x^{3}}=0, \\
& \frac{\partial^{2} f}{\partial x^{1} \partial x^{4}}=0, \\
& \frac{\partial^{2} f}{\partial\left(x^{2}\right)^{2}}-\frac{1}{2} \frac{\partial f}{\partial x^{2}}=(r-\sigma) e^{x^{2}}, \\
& \frac{\partial^{2} f}{\partial x^{2} \partial x^{3}}=0, \\
& \frac{\partial^{2} f}{\partial x^{2} \partial x^{4}}=0, \\
& \frac{\partial^{2} f}{\partial\left(x^{3}\right)^{2}}=(r-\sigma), \\
& \frac{\partial^{2} f}{\partial x^{3} \partial x^{4}}-\cot x^{3} \frac{\partial f}{\partial x^{4}}=0, \\
& \frac{\partial^{2} f}{\partial\left(x^{4}\right)^{2}}+\sin x^{3} \cos x^{3} \frac{\partial f}{\partial x^{3}}=(r-\sigma) \sin ^{2} x^{3} .
\end{aligned}
$$

From (22), (23), (24), (26), and (27), it follows that $f$ is a function of $x^{1}$ only. Then from (28) we have $\sigma=r$ and the relation (25), (29) and (30) are verified. Also from (21), we get

$$
f=2 c e^{\frac{x^{1}}{2}}, c \in I R
$$

This leads to the following:

Theorem 3.2. Let $M$ be a Riemannian manifold endowed with the metric given in (17). Then (M, g) is a Yamabe gradient soliton having $f\left(x^{1}, x^{2}, x^{3}, x^{4}\right)=2 c e^{\frac{x^{1}}{2}}, c \in I R$ as potential function.

Example 3.3. Let $M=\left\{\left(x^{1}, x^{2}, x^{3}, x^{4}\right) \in I R^{4}: x^{3} \neq 0\right\}$ be a manifold endowed with the metric

$$
d s^{2}=g_{i j} d x^{i} d x^{j}=u\left(d x^{1}\right)^{2}+2 d x^{1} d x^{2}+\left(d x^{3}\right)^{2}+\left(d x^{4}\right)^{2},(i, j=1,2,3,4)
$$


International Journal of Mathematical, Engineering and Management Sciences

Vol. 3, No. 4, 491-497, 2018

https://dx.doi.org/10.33889/IJMEMS.2018.3.4-035

where $u=a_{0}+a_{1} x^{3}+e^{x^{1}}\left\{\frac{\left(x^{3}\right)^{2}}{2 !}+\frac{\left(x^{3}\right)^{4}}{4 !}+\frac{\left(x^{3}\right)^{6}}{6 !}+\cdots+\frac{\left(x^{3}\right)^{2 n+2}}{2 n+2 !}+\cdots\right\}, a_{0}, a_{1}$ are non-constant functions of $x^{1}$ only. Then the only non-vanishing components of the $\Gamma_{i j}^{k}, R_{i j k}^{l}, S_{i j}$ and $r$ are given by (Shaikh et al., 2008)

$$
\begin{aligned}
& \Gamma_{11}^{2}=\frac{1}{2} \frac{\partial u}{\partial x^{1}}, \Gamma_{13}^{2}=\frac{1}{2} \frac{\partial u}{\partial x^{3}}=-\Gamma_{11}^{3}, \\
& R_{1331}=\frac{1}{2} \frac{\partial^{2} u}{\partial\left(x^{3}\right)^{2}}=\frac{1}{2} e^{x^{1}} \cosh x^{3}=S_{11,}, \quad r=0 .
\end{aligned}
$$

This manifold $M$ with the metric $g$ in (32) is a Riemannian manifold of vanishing scalar curvature. Also, it may be checked that (Shaikh et al., 2008) it is non-conformally flat. For an arbitrary smooth function $f\left(x^{1}, x^{2}, x^{3}, x^{4}\right)$ on $M$, we compute

$$
\left.\begin{array}{l}
f_{, 11}=\frac{\partial^{2} f}{\partial\left(x^{1}\right)^{2}}-\frac{1}{2} \frac{\partial u}{\partial x^{1}} \frac{\partial f}{\partial x^{1}}+\frac{1}{2} \frac{\partial u}{\partial x^{3}} \frac{\partial f}{\partial x^{3}}, f_{, 12}=\frac{\partial^{2} f}{\partial x^{1} \partial x^{2}}=f_{, 21}, \\
f_{, 13}=\frac{\partial^{2} f}{\partial x^{1} \partial x^{3}}-\frac{1}{2} \frac{\partial u}{\partial x^{3}} \frac{\partial f}{\partial x^{2}}=f_{, 31}, f_{, 14}=\frac{\partial^{2} f}{\partial x^{1} \partial x^{4}}=f_{, 41}, \\
f_{, 22}=\frac{\partial^{2} f}{\partial\left(x^{2}\right)^{2}}, f_{, 23}=\frac{\partial^{2} f}{\partial x^{2} \partial x^{3}}=f_{, 32}, f_{, 24}=\frac{\partial^{2} f}{\partial x^{2} \partial x^{4}}=f_{, 42}, \\
f_{, 33}=\frac{\partial^{2} f}{\partial\left(x^{3}\right)^{2}}, f_{, 34}=\frac{\partial^{2} f}{\partial x^{3} \partial x^{4}}=f_{, 43}, f_{, 44}=\frac{\partial^{2} f}{\partial\left(x^{4}\right)^{2}} .
\end{array}\right\}
$$

In a similar way as previous example, we have from (34), (35) and (1) that

$$
f=\frac{c}{2} e^{x^{1}}\left(\cosh x^{3}-1\right), \quad c \in I R
$$

This leads to the following:

Theorem 3.3. Let $(M, g)$ be a Riemannian manifold equipped with the metric given in (32). Then $(M, g)$ is a Yamabe gradient soliton having potential function $f$ is given by (34).

\section{Conclusion}

In (Cao et al., 2002) Cao et al. introduced the concept of Yamabe gradient soliton. Here we constructed two examples of such soliton on 4-dimensional Riemannian manifold. The first example is a Yamabe gradient soliton with constant potential function, while as the second one is Yamabe gradient soliton with non-constant potential function. In this connection, one can construct the example of such soliton on Riemannian manifold of dimension $n>4$.

Acknowledgement 
International Journal of Mathematical, Engineering and Management Sciences

Vol. 3, No. 4, 491-497, 2018

https://dx.doi.org/10.33889/IJMEMS.2018.3.4-035

The authors would like to express their sincere thanks to the referee and Editor-in-chief for their valuable suggestions towards to the improvement of the paper. The first author (Y. C. Mandal) gratefully acknowledges to the Higher Education Department, Government of West Bengal for financial assistance through Swami Vivekananda Merit Cum Means Scholarship. The second author (S. K. Hui) would like to express his gratitude to the University of Burdwan for providing administrative and technical support.

\section{References}

Bercu, G., \& Postolache, M. (2011). Classes of gradient Ricci solitons. International Journal of Geometric Methods in Modern Physics, 8(4), 783-796.

Calvaruso, G., \& Zaeim, A. (2014). A complete classification of Ricci and Yamabe solitons of nonreductive homogeneous 4-spaces. Journal of Geometry and Physics, 80, 15-25.

Cao, H. D., Sun, X. F., \& Zhang, Y. Y. (2002). On the structure of gradient Yamabe solitons. Mathematical Research Letters, 19, 767-774.

Catino, G., Mantegazza, C., \& Mazzieri, L. (2012). On the global structure of conformal gradient solitons with nonnegative Ricci tensor. Communications in Contemporary Mathematics, 14(6), 12 pages.

Chen, B. Y., \& Deshmukh, S. (2017). Yamabe and quasi-Yamabe solitons on Euclidean submanifolds. arXiv: 1711. 02978.

Daskalopoulos, P., \& Sesum, N. (2013). The classification of locally conformally flat Yamabe solitons. Advances in Mathematics, 240, 346-369.

Di Cerbo, L., \& Disconzi, M. (2008). Yamabe solitons, determinant of the Laplacian and the uniformization theorem for Riemannian surfaces. Letters in Mathematical Physics, 83(1), 13-18.

Erken, K. (2017). Yamabe solitons on three-dimensional normal almost paracontact metric manifolds. arXiv: 1708.04882.

Hamilton, R. S. (1988). The Ricci flow on surfaces, Mathematics and general relativity. Contemporary Mathematics, 71, 237-262.

Hamilton, R. S. (1989). Lectures on geometric flows, unpublished manuscript.

He, C. (2011). Gradient Yamabe solitons on warped products. arXiv:1109. 2343 [math.DG].

Hsu, S. -Y. (2012). A note on compact gradient Yamabe solitons. Journal of Mathematical Analysis and Applications, 388(2), 725-726.

Huang, G., \& Li, H. (2014). On a classification of the quasi Yamabe gradient solitons. Method and Application of Analysis, 21(3), 379-390.

Kundu, S. (2016). On Yamabe soliton. Irish Mathematical Society Bulletin, 77, 51-60.

Shaikh, A. A., Shahid, M. H., \& Hui, S. K. (2008). On weakly conformally symmetric manifolds. Matematicki Vesnik, 60, 269-284.

Sharma, R. (2012). A 3-dimensional Sasakian metric as a Yamabe soliton. International Journal of Geometric Methods in Modern Physics 9(4), 1220003, 5 pages.

Shu, S.-Y. (2011). A note on contact gradient Yamabe solitons. arXiv:1107.3769 [math.DG].

Yamabe, H. (1960). On a deformation of Riemannian structures on compact manifolds. Osaka Mathematical Journal, 12, 21-37. 